\title{
Exponential stability analysis of nonlinear systems with bounded gain error
}

Xingkai $\mathrm{Hu}^{1,2^{*}}$ and Linru $\mathrm{Nie}^{1,2}$

\section{*Correspondence:}

huxingkai84@163.com

${ }^{1}$ Faculty of Civil Engineering and

Mechanics, Kunming University of

Science and Technology, Kunming,

P.R. China

${ }^{2}$ Faculty of Science, Kunming

University of Science and

Technology, Kunming, P.R. China

\begin{abstract}
Considering technology limitation or device restriction in practical application, we formulate new nonlinear systems with bounded gain error, which contain switched control and impulsive control. We then investigate the exponential stability of the considered systems. Finally, the effectiveness of the proposed criteria is confirmed via an example based on Chua's oscillator.
\end{abstract}

MSC: 37 N35; 49N25

Keywords: Exponential stability; Nonlinear systems; Bounded gain error; Impulsive control; Switched control

\section{Introduction}

Nonlinear systems have been paid considerable attention from different areas since they have been successfully used in many practical applications including robotics, information science, artificial intelligence, automatic control systems, and so forth [1,2]. Due to various effects, the states of systems will become oscillations and instability. Thus, it is significant to discuss stability of nonlinear systems. There are many methods to stabilize the nonlinear systems, for example, adaptive control [3], fuzzy control [4], sliding mode control [5], feedback control [6], impulsive control [7], switched control [8], etc. In view of engineering applications, the control cost of continuous control is expensive. By intermittent control, control cost and the amount of the transmitted information can be reduced drastically. It should be noticed that both impulsive control and switched control are discontinuous control methods.

Impulsive control of nonlinear systems has been one of the focal points in many research and application fields, such as complex networks, orbital transfer of satellite, dosage supply in pharmacokinetics, ecosystems management, synchronization in chaotic secure communication systems [9-15], etc. Impulsive control can stabilize nonlinear systems by using it only at some isolated points.

Switched control system is a hybrid system that is composed of several subsystems and a switching rule that orchestrates the switching among subsystems. In the real world, many biological, physical, engineering, and economical systems can be presented by switched systems. Compared with ordinary differential dynamic systems, not only should we focus on each subsystem, but the switching rule as well. Switching among different subsystems can cause chaos and instability. It is well known that a switched system might be stable

(c) The Author(s) 2019. This article is distributed under the terms of the Creative Commons Attribution 4.0 International License (http://creativecommons.org/licenses/by/4.0/), which permits unrestricted use, distribution, and reproduction in any medium, provided you give appropriate credit to the original author(s) and the source, provide a link to the Creative Commons license, and indicate if changes were made. 
even if each subsystem is unstable and also might be unstable even if all subsystems are stable for specified switching rules.

In this paper, switched control and impulsive control are combined together. We construct a control system, in which some of the inputs are continuous and some are impulsive.

The remainder of this paper is organized as follows. The considered model of general nonlinear systems with bounded gain error is given in Sect. 2. Some necessary notations and lemmas are also presented in this section. In Sect. 3, we establish an exponential stability criterion. Then, in Sect. 4, an example is presented to show the effectiveness of our result. Finally, we conclude the paper.

\section{Problem formulation and preliminaries}

A class of nonlinear systems can be described as

$$
\left\{\begin{array}{l}
\dot{x}(t)=A x(t)+f(x(t))+w(t) \\
x\left(t_{0}\right)=x_{0}
\end{array}\right.
$$

where $x(t) \in R^{n}$ is the state vector, $A \in R^{n \times n}$ is a constant matrix, $f: R^{n} \rightarrow R^{n}$ is a continuous nonlinear function satisfying $f(0)=0$, and $\|f(x)\| \leq l\|x\|, l \geq 0$ is a constant. $w(t)$ is control input. Without loss of generally, let $t_{0}=0, x_{0} \in R^{n}$ is a given vector.

In order to stabilize system (2.1) at the origin, we set three kinds of control, i.e., in the first period of continuous time, we set $w(t)=B_{1} x(t)$, where $B_{1} \in R^{n \times n}$ is a known matrix; in the second period of continuous time, we set $w(t)=B_{2} x(t)$, where $B_{2} \in R^{n \times n}$ is a known matrix; at the same time, where the system is changed from the first control to the second control, we impose an impulse.

So system (2.1) is rewritten as

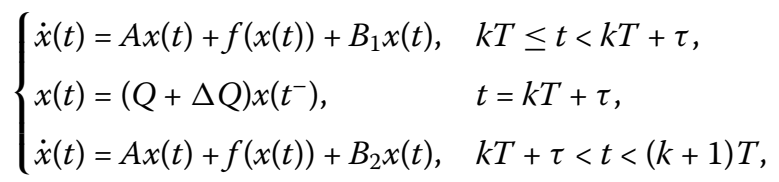

where $T>0$ represents control period, $\tau \in(0, T)$ is a constant. $Q \in R^{n \times n}$ is the impulsive control gain matrix, $\Delta Q \in R^{n \times n}$ denotes impulsive gain error caused by technology limitation or device restriction. In general, let

$$
\Delta Q=m G(t) Q
$$

where $m$ is a positive constant. The uncertain matrix $G(t) \in R^{n \times n}$ satisfies

$$
G^{T}(t) G(t) \leq I
$$

Remark 1 Recently, periodic control has been extensively investigated, intermittent and alternate cases have been separately studied. To unify them, the generalized control protocol (2.2) is presented in this paper, which contains the traditional periodically intermittent control [16], periodically alternate control $[17,18]$, and impulsive control. 
Lemma 1 ([19]) Let $x, y \in R^{n}$, then

$$
\left|x^{T} y\right| \leq\|x\|\|y\| .
$$

Lemma 2 ([20]) Let $x, y \in R^{n}$ and $\varepsilon>0$, then

$$
2 x^{T} y \leq \varepsilon x^{T} x+\frac{1}{\varepsilon} y^{T} y .
$$

Lemma 3 ([19]) Suppose that $A \in R^{n \times n}$ is a symmetric matrix. Then, for all $x \in R^{n}$,

$$
\lambda_{\min }(A) x^{T} x \leq x^{T} A x \leq \lambda_{\max }(A) x^{T} x .
$$

As customary, $R^{n}$ is an $n$-dimensional real Euclidean space with norm $\|\cdot\| \cdot R^{m \times n}$ denotes the set of all $m \times n$-dimensional real matrices. $\lambda_{\min }(A), \lambda_{\max }(A)$, and $A^{T}$ are the minimum, the maximum eigenvalue, and the transpose of matrix $A$, respectively. $A>0$ implies that $A$ is a positive definite matrix. $I$ is an identity matrix of proper dimension. $f\left(x\left(t_{0}^{-}\right)\right)$is defined by $f\left(x\left(t_{0}^{-}\right)\right)=\lim _{t \rightarrow t_{0}^{-}} f(x(t))$.

\section{Stability analysis}

In this section, we aim at proposing the exponential stability criterion of system (2.2).

Theorem 1 Let $0<P \in R^{n \times n}$ such that the following two conditions are satisfied:

(1) $h_{1}<0$,

(2) $h_{1} \tau+h_{2}(T-\tau)+\ln \eta<0$,

where $\beta_{1}=\lambda_{\max }\left(P^{-1}\left(P A+A^{T} P+P B_{1}+B_{1}^{T} P\right)\right), \quad \beta_{2}=\lambda_{\max }(P), \quad \beta_{3}=\lambda_{\min }(P), \quad \beta_{4}=$ $\lambda_{\max }\left(P^{-1}\left(Q^{T} Q\right)\right), \beta_{5}=\lambda_{\max }\left(P^{-1}\left(P A+A^{T} P+P B_{2}+B_{2}^{T} P\right)\right), h_{1}=\beta_{1}+2 l \sqrt{\frac{\beta_{2}}{\beta_{3}}}, \eta=\beta_{2} \beta_{4}((1+$ $\left.\varepsilon)+m^{2}\left(1+\frac{1}{\varepsilon}\right)\right), h_{2}=\beta_{5}+2 l \sqrt{\frac{\beta_{2}}{\beta_{3}}}$. Then system (2.2) is exponentially stable at origin.

Proof Define

$$
V(x(t))=x^{T}(t) P x(t)
$$

Let $t \in[k T, k T+\tau)$, from Lemmas 1 and 3 , we obtain

$$
\begin{aligned}
D^{+}(V(x(t))) & =2 x^{T}(t) P\left(A x(t)+f(x(t))+B_{1} x(t)\right) \\
& =2 x^{T}(t) P A x(t)+2 x^{T}(t) P f(x(t))+2 x^{T}(t) P B_{1} x(t) \\
& =x^{T}(t)\left(P A+A^{T} P+P B_{1}+B_{1}^{T} P\right) x(t)+2 x^{T}(t) P^{\frac{1}{2}} P^{\frac{1}{2}} f(x(t)) \\
& \leq \beta_{1} x^{T}(t) P x(t)+2 \sqrt{x^{T}(t) P x(t) f^{T}(x(t)) P f(x(t))} \\
& \leq \beta_{1} x^{T}(t) P x(t)+2 \sqrt{x^{T}(t) P x(t) \beta_{2} f^{T}(x(t)) f(x(t))} \\
& \leq \beta_{1} x^{T}(t) P x(t)+2 \sqrt{x^{T}(t) P x(t) \beta_{2} l^{2} x^{T}(t) x(t)} \\
& \leq \beta_{1} x^{T}(t) P x(t)+2 l \sqrt{x^{T}(t) P x(t) \frac{\beta_{2}}{\beta_{3}} x^{T}(t) P x(t)}
\end{aligned}
$$




$$
=h_{1} V(x(t)) \text {, }
$$

which means

$$
V(x(t)) \leq V\left(x(k T)^{-}\right) e^{h_{1}(t-k T)} .
$$

If $t=k T+\tau$, then from Lemmas 2 and 3 we have

$$
\begin{aligned}
V(x(t)) & =\left((Q+\Delta Q) x\left(t^{-}\right)\right)^{T} P(Q+\Delta Q) x\left(t^{-}\right) \\
& =x^{T}\left(t^{-}\right)\left(Q^{T} P Q+Q^{T} P \Delta Q+\Delta Q^{T} P Q+\Delta Q^{T} P \Delta Q\right) x\left(t^{-}\right) \\
& \leq x^{T}\left(t^{-}\right)\left((1+\varepsilon) Q^{T} P Q+\left(1+\frac{1}{\varepsilon}\right) \Delta Q^{T} P \Delta Q\right) x\left(t^{-}\right) \\
& \leq \beta_{2} x^{T}\left(t^{-}\right)\left((1+\varepsilon) Q^{T} Q+\left(1+\frac{1}{\varepsilon}\right) \Delta Q^{T} \Delta Q\right) x\left(t^{-}\right) \\
& =\beta_{2} x^{T}\left(t^{-}\right)\left((1+\varepsilon) Q^{T} Q+m^{2}\left(1+\frac{1}{\varepsilon}\right) Q^{T} G^{T}(t) G(t) Q\right) x\left(t^{-}\right) \\
& \leq \beta_{2} x^{T}\left(t^{-}\right)\left((1+\varepsilon) Q^{T} Q+m^{2}\left(1+\frac{1}{\varepsilon}\right) Q^{T} Q\right) x\left(t^{-}\right) \\
& \leq \beta_{2} \beta_{4}\left((1+\varepsilon)+m^{2}\left(1+\frac{1}{\varepsilon}\right)\right) V\left(x\left(t^{-}\right)\right) \\
& =\eta V\left(x\left(t^{-}\right)\right) .
\end{aligned}
$$

In the same way, let $t \in(k T+\tau,(k+1) T)$, we also obtain

$$
\begin{aligned}
D^{+}(V(x(t))) & =2 x^{T}(t) P\left(A x(t)+f(x(t))+B_{2} x(t)\right) \\
& =x^{T}(t)\left(P A+A^{T} P+P B_{2}+B_{2}^{T} P\right) x(t)+2 x^{T}(t) P f(x(t)) \\
& \leq \beta_{5} x^{T}(t) P x(t)+2 \sqrt{x^{T}(t) P x(t) f^{T}(x(t)) P f(x(t))} \\
& \leq h_{2} V(x(t)),
\end{aligned}
$$

which together with (3.2) infers that

$$
V(x(t)) \leq \eta V\left(x(k T+\tau)^{-}\right) e^{h_{2}(t-k T-\tau)},
$$

where $t \in[k T+\tau,(k+1) T)$.

When $k=0$, let $t \in[0, \tau)$, from (3.1) we can obtain

$$
V(x(t)) \leq V(x(0)) e^{h_{1} t},
$$

hence

$$
V\left(x\left(\tau^{-}\right)\right) \leq V(x(0)) e^{h_{1} \tau} .
$$


Let $t \in[\tau, T)$, applying (3.3) and (3.4), we get

$$
\begin{aligned}
V(x(t)) & \leq \eta V\left(x\left(\tau^{-}\right)\right) e^{h_{2}(t-\tau)} \\
& \leq \eta V(x(0)) e^{h_{1} \tau+h_{2}(t-\tau)},
\end{aligned}
$$

hence

$$
V\left(x\left(T^{-}\right)\right) \leq \eta V(x(0)) e^{h_{1} \tau+h_{2}(T-\tau)} .
$$

When $k=1$, let $t \in[T, T+\tau)$, applying (3.1) and (3.5), we get

$$
\begin{aligned}
V(x(t)) & \leq V\left(x\left(T^{-}\right)\right) e^{h_{1}(t-T)} \\
& \leq \eta V(x(0)) e^{h_{1} \tau+h_{2}(T-\tau)+h_{1}(t-T)},
\end{aligned}
$$

hence

$$
V\left(x\left((T+\tau)^{-}\right)\right) \leq \eta V(x(0)) e^{2 h_{1} \tau+h_{2}(T-\tau)} .
$$

Let $t \in[T+\tau, 2 T)$, applying (3.3) and (3.6), we get

$$
\begin{aligned}
V(x(t)) & \leq \eta V\left(x\left((T+\tau)^{-}\right)\right) e^{h_{2}(t-T-\tau)} \\
& \leq \eta^{2} V(x(0)) e^{2 h_{1} \tau+h_{2}(T-\tau)+h_{2}(t-T-\tau)} .
\end{aligned}
$$

By induction, when $k=m, m=0,1, \ldots$, let $t \in[m T, m T+\tau)$, we get

$$
V(x(t)) \leq \eta^{m} V(x(0)) e^{m h_{1} \tau+m h_{2}(T-\tau)+h_{1}(t-m T)},
$$

hence

$$
V\left(x\left((m T+\tau)^{-}\right)\right) \leq \eta^{m} V(x(0)) e^{(m+1) h_{1} \tau+m h_{2}(T-\tau)} .
$$

Let $t \in[m T+\tau,(m+1) T)$, applying (3.3) and (3.8), we obtain

$$
\begin{aligned}
V(x(t)) & \leq \eta V\left(x\left((m T+\tau)^{-}\right)\right) e^{h_{2}(t-m T-\tau)} \\
& \leq \eta^{m+1} V(x(0)) e^{(m+1) h_{1} \tau+m h_{2}(T-\tau)+h_{2}(t-m T-\tau)} .
\end{aligned}
$$

Applying (3.7), we get

$$
\begin{aligned}
V(x(t)) & \leq \eta^{m} V(x(0)) e^{m h_{1} \tau+m h_{2}(T-\tau)} \\
& =V(x(0)) e^{m\left(h_{1} \tau+h_{2}(T-\tau)+\ln \eta\right)} \\
& <V(x(0)) e^{\frac{t-\tau}{T}\left(h_{1} \tau+h_{2}(T-\tau)+\ln \eta\right)} \\
& <V(x(0)) e^{\frac{t-T}{T}\left(h_{1} \tau+h_{2}(T-\tau)+\ln \eta\right)},
\end{aligned}
$$

where $t \in[m T, m T+\tau)$. 
Let $t \in[m T+\tau,(m+1) T)$, applying (3.9), we get the following.

Case 1 . When $h_{2}>0$, we get

$$
\begin{aligned}
V(x(t)) & <\eta^{m+1} V(x(0)) e^{(m+1) h_{1} \tau+(m+1) h_{2}(T-\tau)} \\
& <V(x(0)) e^{\frac{t}{T}\left(h_{1} \tau+h_{2}(T-\tau)+\ln \eta\right)} \\
& <V(x(0)) e^{\frac{t-T}{T}\left(h_{1} \tau+h_{2}(T-\tau)+\ln \eta\right)}
\end{aligned}
$$

Case 2. When $h_{2} \leq 0$, we get

$$
\begin{aligned}
V(x(t)) & \leq \eta^{m+1} V(x(0)) e^{(m+1) h_{1} \tau+m h_{2}(T-\tau)} \\
& <\eta^{m+1} V(x(0)) e^{m h_{1} \tau+m h_{2}(T-\tau)} \\
& <\eta V(x(0)) e^{\frac{t-T}{T}\left(h_{1} \tau+h_{2}(T-\tau)+\ln \eta\right)} .
\end{aligned}
$$

For all $t>0$, by (3.10), (3.11), and (3.12), we can conclude that system (2.2) is exponentially stable at origin.

This completes the proof.

\section{A numerical example}

For verifying the effectiveness of Theorem 1, a numerical example is presented in this section.

Example 1 Chua's oscillator [21] is given by

$$
\left\{\begin{array}{l}
\dot{x}_{1}=\alpha\left(x_{2}-x_{1}-g\left(x_{1}\right)\right) \\
\dot{x}_{2}=x_{1}-x_{2}+x_{3} \\
\dot{x}_{3}=-\gamma x_{2}
\end{array}\right.
$$

where $\alpha$ and $\gamma$ are parameters,

$$
g\left(x_{1}\right)=b x_{1}+0.5(a-b)\left(\left|x_{1}+1\right|-\left|x_{1}-1\right|\right),
$$

where $a<b<0$ are two given constants.

For using the above result, system (4.1) is rewritten as

$$
\dot{x}(t)=A x+f(x)
$$

where

$$
\begin{aligned}
& A=\left(\begin{array}{ccc}
-\alpha(1+b) & \alpha & 0 \\
1 & -1 & 1 \\
0 & -\gamma & 0
\end{array}\right) \\
& f(x)=\left(\begin{array}{c}
-0.5 \alpha(a-b)\left(\left|x_{1}+1\right|-\left|x_{1}-1\right|\right) \\
0 \\
0
\end{array}\right) .
\end{aligned}
$$




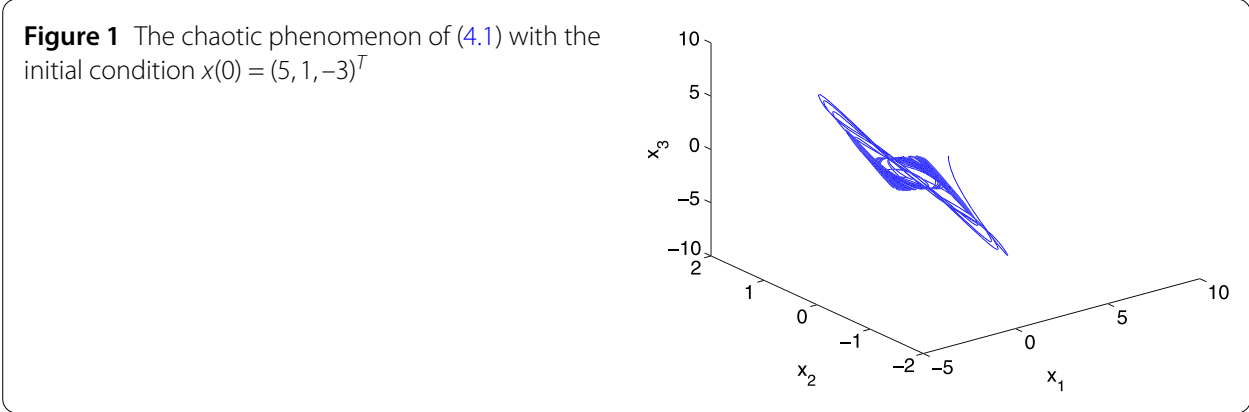

Figure 2 Time response curves of (4.1) with the initial condition $x(0)=(5,1,-3)^{\top}$

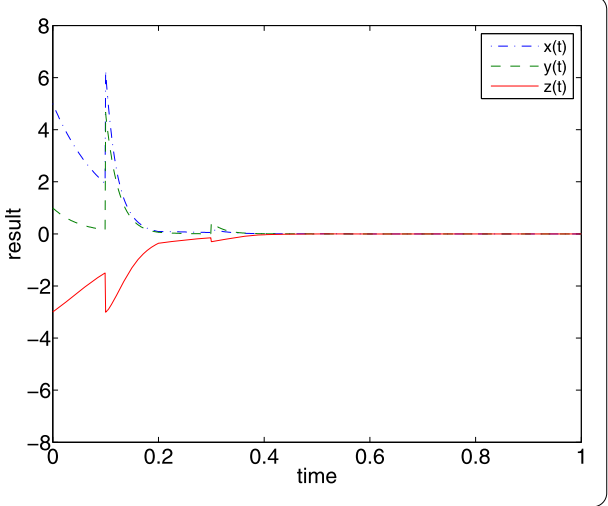

In the initial condition $x(0)=(5,1,-3)^{T}$, system (4.1) has chaotic phenomenon when

$$
\alpha=9.2156, \quad \gamma=15.9946, \quad a=-1.24905, \quad b=-0.75735,
$$

as shown in Fig. 1. In this case, we can get $l=4.5313$.

In order to simplify the calculation, let $P=I, \tau=0.1, T=0.2, \varepsilon=0.5, m=1, B_{1}=$ $\operatorname{diag}(-10,-20,-10), B_{2}=\operatorname{diag}(-50,-40,-30)$, and

$$
Q=\left[\begin{array}{ccc}
3 & 3 & 0 \\
0 & 1 & -3 \\
0 & 0 & 2
\end{array}\right] .
$$

Through simple computation, we get $\beta_{1}=-10.5898, \beta_{2}=\beta_{3}=1, \beta_{4}=8.21, \beta_{5}=-51.9794$, $h_{1}=-1.5272<0, h_{2}=-42.9168, \eta=36.945$, and $h_{1} \tau+h_{2}(T-\tau)+\ln \eta=-0.7630<0$. So, system (4.1) is exponentially stable by Theorem 1 with the initial condition $x(0)=$ $(5,1,-3)^{T}$, as shown in Fig. 2.

\section{Conclusions}

In this paper, we investigate the exponential stability of nonlinear systems with bounded gain error. A generalized control model, which contains switched control and impulsive control, is introduced. Compared with the traditional periodic control [16-18], system (2.2) is more general and more practical. Finally, a numerical example is given to show the effectiveness of the proposed method. 
Acknowledgements

The authors would like to express their sincere thanks to referees and the editor for their enthusiastic guidance and help.

\section{Funding}

This research is supported by the National Natural Science Foundation of China (Grant Nos. 11561037, 11661047, 11801240).

\section{Availability of data and materials}

Not applicable.

\section{Competing interests}

The authors declare that they have no competing interests.

\section{Authors' contributions}

The authors contributed equally to the manuscript. Both authors read and approved the final manuscript.

\section{Publisher's Note}

Springer Nature remains neutral with regard to jurisdictional claims in published maps and institutional affiliations.

Received: 19 September 2019 Accepted: 6 November 2019 Published online: 14 November 2019

\section{References}

1. Lakshmikantham, V., Bainov, D.D., Simeonov, P.S.: Theory of Impulsive Differential Equations. World Scientific, Singapore (1989)

2. Yang, T.: Impulsive Control Theory. Springer, Berlin (2001)

3. He, W., Meng, T.: Adaptive control of a flexible string system with input hysteresis. IEEE Trans. Control Syst. 26, 693-700 (2018)

4. Pan, Y., Yang, G.: A novel event-based fuzzy control approach for continuous-time fuzzy systems. Neurocomputing 338, 55-62 (2019)

5. He, S., Song, J., Liu, F.: Robust finite-time bounded controller design of time-delay conic nonlinear systems using sliding mode control strategy. IEEE Trans. Syst. Man Cybern. 48, 1863-1873 (2018)

6. Cardinali, T., Rubbioni, P.: The controllability of an impulsive integro-differential process with nonlocal feedback controls. Appl. Math. Comput. 347, 29-39 (2019)

7. Li, X., Song, S.: Stabilization of delay systems: delay-dependent impulsive control. IEEE Trans. Autom. Control 62 406-411 (2017)

8. Xu, H., Teo, K.: Exponential stability with L2-gain condition of nonlinear impulsive switched systems. IEEE Trans. Autom. Control 55, 2429-2433 (2010)

9. Huang, T., Li, C., Duan, S., Starzyk, J.A.: Robust exponential stability of uncertain delayed neural networks with stochastic perturbation and impulse effects. IEEE Trans. Neural Netw. Learn. Syst. 23, 866-875 (2012)

10. Yang, X., Lu, J.: Finite-time synchronization of coupled networks with Markovian topology and impulsive effects. IEEE Trans. Autom. Control 61, 2256-2261 (2016)

11. Lu, J., Ding, C., Lou, J., Cao, J.: Outer synchronization of partially coupled dynamical networks via pinning impulsive controllers. J. Franklin Inst. 352, 5024-5041 (2015)

12. Li, X., Ho, D.W.C., Cao, J.: Finite-time stability and settling-time estimation of nonlinear impulsive systems. Automatica 99, 361-368 (2019)

13. Li, X., Yang, X., Huang, T.: Persistence of delayed cooperative models: impulsive control method. Appl. Math. Comput. $342,130-146(2019)$

14. Sun, J., Zhang, Y., Wu, Q.: Impulsive control for the stabilization and synchronization of Lorenz systems. Phys. Lett. A 298, 153-160 (2002)

15. Zou, L., Peng, Y., Feng, Y., Tu, Z.: Stabilization and synchronization of memristive chaotic circuits by impulsive control. Complexity 2017, Article ID 5186714 (2017)

16. Li, C., Feng, G., Liao, X.: Stabilization of nonlinear systems via periodically intermittent control. IEEE Trans. Circuits Syst. II, Express Briefs 54, 1019-1023 (2007)

17. Feng, Y., Li, C., Huang, T., Zhao, W.: Alternate control systems. Adv. Differ. Equ. 2014, 305 (2014)

18. Hu, X., Nie, L.: Exponential stability of nonlinear systems via alternate control. Ital. J. Pure Appl. Math. 40, 671-678 (2018)

19. Horn, R.A., Johnson, C.R.: Matrix Analysis. Cambridge University Press, Cambridge (1985)

20. Zou, L., Peng, Y., Feng, Y., Tu, Z.: Impulsive control of nonlinear systems with impulse time window and bounded gain error. Nonlinear Anal., Model. Control 23, 40-49 (2018)

21. Shilnikov, L.P.: Chau's circuit: rigorous results and future problems. Int. J. Bifurc. Chaos Appl. Sci. Eng. 4, 489-519 (1994) 